\title{
First-Night Effect on Sleep in Different Female Reproductive States
}

\author{
Virtanen, Irina
}

2018

Virtanen , I , Kalleinen , N , Urrila , A S \& Polo-Kantola , P 2018 , ' First-Night Effect on Sleep in Different Female Reproductive States ', Behavioral sleep medicine , vol. 16 , no. 5 , pp. 437-447 . https://doi.org/10.1080/15402002.2016.1228646

http://hdl.handle.net/10138/310855

https://doi.org/10.1080/15402002.2016.1228646

acceptedVersion

Downloaded from Helda, University of Helsinki institutional repository.

This is an electronic reprint of the original article.

This reprint may differ from the original in pagination and typographic detail.

Please cite the original version. 


\section{FIRST NIGHT EFFECT ON SLEEP IN DIFFERENT FEMALE REPRODUCTIVE STATES}

Running head: First night effect in women

Irina Virtanen, $\mathrm{MD}, \mathrm{PhD}^{1,2} *$, Nea Kalleinen, $\mathrm{MD}, \mathrm{PhD}^{2,3}$, Anna $\mathrm{S}$. Urrila, $\mathrm{MD}, \mathrm{PhD}^{4,5}$, and Päivi Polo-Kantola, $\mathrm{MD}, \mathrm{PhD}^{2,6}$

1 Department of Clinical Neurophysiology, TYKS-SAPA, Hospital District of Southwest Finland, Turku, Finland

2 Sleep Research Unit, University of Turku, Turku, Finland

3 Department of Cardiology, Satakunta Central Hospital, Pori, Finland

4 Department of Health, Mental Health Unit, National Institute for Health and Welfare, Helsinki, Finland

5 Department of Adolescent Psychiatry, Helsinki University Hospital, Helsinki, Finland 6 Department of Obstetrics and Gynecology, Turku University Hospital and University of Turku, Turku, Finland

Correspondence to:

Irina Virtanen, $\mathrm{MD}, \mathrm{PhD}$

Department of Clinical Neurophysiology

TYKS-SAPA

Hospital District of Southwest Finland

Kiinamyllynkatu 4-8

PB 52

20521 Turku

irina.virtanen@tyks.fi 


\begin{abstract}
Objectives: In sleep laboratory studies, the new environment is generally considered to disturb sleep during the first night. However, older women have rarely been studied. Although menopause and hormone therapy affect sleep, their effect on the first night effect is virtually unknown.
\end{abstract}

Participants: Four groups of women with no sleep laboratory experience: young on hormonal contraceptives $(n=11,23.1(0.5)$ years $)$, perimenopausal $(n=15,48.0(0.4)$ years $)$, postmenopausal without hormone therapy (HT) (off-HT, n=22, $63.4(0.8)$ years) and postmenopausal with HT ( $\mathrm{n}=16,63.1$ (0.9) years).

Procedure: A cross-sectional study.

Methods: Polysomnography was performed over two consecutive nights and the first night effect and group differences were evaluated. Questionnaire-based insomnia and sleepiness scores were correlated to sleep variables and their between-night changes.

Results: Although sleep in young women was deeper and less fragmented than in the other groups, first night effect was similar in all study groups. Total sleep time, sleep efficiency, and S1 and S2 sleep increased, and wake after sleep onset, awakenings per hour of sleep, S2 and REM latencies, and percentage of SWS decreased from the first to the second night. Perimenopausal women had more insomnia complaints than other women. Insomnia complaints were associated with more disturbed sleep but not with the first night effect. Conclusions: A first night in a sleep laboratory elicits a marked interference of sleep architecture in women of all ages, with a carry-over effect of lighter sleep on the second study night. Menopausal state, HT use or insomnia complaints do not modify this effect. 


\section{Introduction}

The first night effect, i.e. disturbed sleep during the first laboratory polysomnography (PSG) night, was first described by Agnew et al (1966). They observed more awake periods, less rapid eye movement (REM) sleep, a delay in the onset of both slow wave sleep (SWS) and REM sleep, and an increase in sleep stage transitions during a first laboratory night. In healthy adults and elderly people, literature is consistent that sleep efficiency (SE), total sleep time (TST), and the amounts of SWS and REM sleep are decreased, sleep latency (SL) and REM latency are prolonged, and light sleep (S1 / N1) and wake time are increased during a first night in a sleep laboratory (Curcio, Ferrara, Piergianni, Fratello, \& De Gennaro, 2004; Kis et al., 2014; Newell, Mairesse, Verbanck, \& Neu, 2012; Toussaint et al., 1995; Webb \& Campbell, 1979; Zheng et al., 2012) or even in PSG recordings conducted at home (Wauquier, van Sweden, Kerkhof, \& Kamphuisen, 1991). The first night effect is crucial especially in studies investigating the effects of e.g. different medications or sleep deprivation on PSG parameters. In such studies comparative baseline night before the interventions is needed which should be free from disturbances like being unaccustomed to sleep environment. It has been suggested that the first night effect may actually last for several nights (Le Bon et al., 2001), but a study by Lorenzo and Barbanoj ( 2002) proposed that the first night effect is literally confined to the first night in a sleep laboratory.

Regardless of a fairly large database, only two studies have focused on first night effect exclusively in women. Webb and Campbell ( 1979) observed a more pronounced first night effect in older women, but they did not define their reproductive state. The Study of Women's Health Across the Nation (SWAN) (Zheng et al., 2012) studied groups of peri- and postmenopausal women and found shorter TST, lower SE, less REM sleep and delta activity, a longer SL and more wake after sleep onset (WASO) during the first night. They did not 
include young, fertile women or HT users and therefore, information on the influence of different female reproductive states on first night effect is lacking. Studying the first night effect in different female reproductive states is justified given that sleep quality in women deteriorates with age (Kalleinen et al., 2006), and many studies show that sleep quality is worse in the perimenopause and postmenopause, especially in women with climacteric vasomotor symptoms (Lampio et al., 2014; Polo-Kantola, 2011; Shaver, Giblin, Lentz, \& Lee, 1988; Young, Rabago, Zgierska, Austin, \& Finn, 2003). HT is the first line treatment for climacteric vasomotor symptoms (de Villiers et al., 2013) and thus sleep, especially insomnia symptoms, also improves during HT (Polo-Kantola, 2011). Furthermore, HT has been shown to ameliorate the sleep disruption elicited by nocturnal blood sampling, which can be compared to the disturbance elicited by PSG electrodes in study-naïve individuals (Moe, Larsen, Vitiello, \& Prinz, 2001).

Based on the above rationale, we set up to investigate the first night effect and its possible differences in female reproductive states from early adulthood to late postmenopause, with the addition of the effect of HT. We hypothesized that the changes in hormonal levels due to menopause and the use of HT in postmenopause could influence the ability to adapt to a foreign sleeping environment.

\section{Methods}

\section{Participants}

The study was a part of a larger study exploring the effects of ageing, hormonal state and HT on sleep and cognition. The study was performed in the Universities of Turku and Helsinki, Finland. The Turku Sleep Research Unit served as the supervising and monitoring unit of the study. Perimenopausal and postmenopausal women were recruited from the Turku area 
through newspaper announcements, and young women from Helsinki by announcement at the university. Exclusion criteria consisted of existing cardiovascular (hypertension controlled with medication was accepted), pulmonary, neurological, endocrinological or mental disease, irregular or delayed sleep schedule and specific sleep disorders, such as sleep apnea, narcolepsy or restless legs syndrome. Women suffering from conditions that could affect sleep, like fibromyalgia and anemia, were excluded. Other exclusion criteria included the use of medication affecting the central nervous system, including sleeping pills, alcohol abuse, smoking and excessive caffeine intake (over 5 cups of coffee per day). Starting one week before the study, caffeine and alcohol use was prohibited. Before the study, blood hemoglobin, leucocytes, thrombocytes and serum thyrotropin levels were measured and required to fall within normal ranges. Regular medication was used by 15 peri- and postmenopausal women: four women used salicylates, five used statins and ten used antihypertensive medications (beta blockers, calcium blockers, AT1-receptor antagonists, or diuretics). None of the study subjects had sleep laboratory experience.

Information about the study and screening for exclusion criteria was given during a 10-30 min telephone interview (P.P-K., N.K.). Women meeting inclusion criteria were invited for a clinical interview and physical examination (duration 1-1.5 h, P.P-K.) in which exclusion criteria were re-screened and study information was provided. Mood disorders were excluded by filling in the Beck Depression Inventory (BDI). The study protocol is described in detail in previous reports by Kalleinen et al. $(2008 ; 2006)$. The women kept a sleep diary three weeks before and one week after the sleep studies to ensure a regular sleep-wake schedule required for inclusion (from 22:00-23:00 hr to 06:00-07:00 hr). Before the study, the participants also filled in the validated and widely used Basic Nordic Sleep Questionnaire (BNSQ) (Partinen \& Gislason, 1995), from which the sums of insomnia and sleepiness scores were calculated. 
Climacteric symptomatology was scored before the first night with questions on the frequency of night sweats and hot flashes during the last six months in a scale of 1 to 4 ( $1=$ seldom or never, $2=$ once a month, $3=$ once a week, $4=$ almost every day). No group differences in symptom scores were observed. Background information is shown in Table 1.

Of those recruited to the study, 17 were perimenopausal, 44 postmenopausal and 11 young women. Women were defined as perimenopausal if serum FSH levels were lower than 23 IU/L and they had ongoing regular or irregular menstrual cycle, whereas postmenopausal women were defined by age over 57 years and chronic amenorrhea of more than one year. Perimenopausal women [aged 47.7 (0.5) years] did not use HT. Sixteen postmenopausal women [aged $63.1(0.9)$ years] used HT (on-HT group, oral n=10, continuous combined, estradiol hemihydrate $2 \mathrm{mg}+$ norethisterone acetate $1 \mathrm{mg}$ or transdermal $\mathrm{n}=6,17 \beta$-estradiol $50 \mu \mathrm{g} / 24 \mathrm{~h}+$ norethisterone acetate $250 \mathrm{mg} / 24 \mathrm{~h}$ patch), for a mean time of 12.0 (1.2) years. Twenty-eight postmenopausal women had either never used HT $(n=8)$ or had a time since discontinuation of at least 12 months with 5.7 (1.0) years of previous use [off-HT group, aged 63.9 (0.8) years]. In both on-HT and off-HT groups, three women had undergone surgical menopause (minimum six years prior to the study). Young women [aged $23.1(0.5)$ years] used oral contraceptives (ethinyl estradiol $20 \mu \mathrm{g}+$ desogestrel $0.15 \mathrm{mg}$ ). Perimenopausal and young women were examined during the first days of their menstrual cycle. Due to insufficient data, we were able to analyse recordings from 10 young women [aged $23.0(0.5)$ years], 15 perimenopausal women [aged $48.0(0.4)$ years], 22 postmenopausal women off-HT [aged $63.4(0.8)$ years], and 14 postmenopausal women on-HT [aged 63.2 (0.9) years]. 
For plasma follicle stimulating hormone $(\mathrm{FSH})$ and serum estradiol $\left(\mathrm{E}_{2}\right)$ measurements, a blood sample was drawn in the morning following the first night to ensure appropriate use of HT. Height and weight were measured and body mass index (BMI) was calculated accordingly. The Sleep Research Unit provided pre-arranged, nutritionally similar meals and caffeine free coffee or tea served at specific times for the subjects during the study.

PSG was performed over two consecutive nights from $11 \mathrm{pm}$ to $7 \mathrm{am}$. In all women, the PSG recordings comprised continuous monitoring of two electroencephalograms (EEG, C3/A2, C4/A1), two electro-oculograms (EOG), a mandibular electromyogram (EMG) and an electrocardiogram (ECG) with a sampling frequency of $200 \mathrm{~Hz}$. In addition, the perimenopausal and postmenopausal women had two additional EEG channels (O1/A2, O2/A1). (Embla, Medcare Flaga hf. Medical devices, Reykjavik, Iceland). The mean analysed time was $478.9 \pm 0.9$ min during first nights, and $479.9 \pm 0.2 \mathrm{~min}$ during second nights $(\mathrm{p}=\mathrm{ns})$. All recordings were visually double-scored in $30 \mathrm{~s}$ epochs off-line by experienced scorers (N.K., P.P.-K. and I.V.) according to the Rechtschaffen and Kales criteria (1968) valid during data collection (years 2001-2004). Three non-REM (NREM) sleep stages [stage 1 (S1), stage 2 (S2), SWS (stages 3 and 4 combined)] and REM sleep, as well as wake and movement time (MT), were classified and expressed as minutes and per cent of total sleep time (TST). Additionally, SE (TST as per cent of total recording time), SL (from lights off to sleep onset, defined as the appearance of three consecutive epochs of S1 or the first epoch of any other stage), latency to the first 30 seconds of S2, SWS and REM sleep from sleep onset, wake after sleep onset (WASO) and awakening index (the number of awakenings per hour of sleep) were calculated. 
Statistical analyses were made using the IBM SPSS ${ }^{\circledR}$ ver. 22.0 software. Data were first tested for normality and observed to be highly skewed, and accordingly, differences between groups in sleep variables were analysed at both nights and for the first night effect using the independent samples Kruskall-Wallis test. Post-hoc analyses were made using the MannWhitney U-test with Bonferroni correction. First night effect was then analysed both using combined groups and group-wise using the Wilcoxon signed rank test. Spearman correlation coefficients were calculated between the BNSQ insomnia and sleepiness scores, vasomotor symptom scores, serum FSH levels, and the objective sleep variables during both nights. To tease out a possible reverse first night effect and hormonal effects, same correlations were applied to inter-night differences in sleep variables. All results are expressed as mean (SEM). $\mathrm{P}$ values of $<0.05$ were considered significant.

\section{Ethics}

The study was approved by the Ethical Committees of Turku University Central Hospital and University of Helsinki. All the study participants gave their written informed consent.

\section{Results}

Group differences on first night

During the first night, young women had less S1 and more SWS than other groups. They also had a longer TST, less WASO, and a lower awakening index than the postmenopausal groups. Furthermore, young women had less wake time, a higher SE and more REM sleep than the off-HT group. Perimenopausal women had more REM sleep than postmenopausal off-HT women. Between postmenopausal groups, off-HT women had more SWS than on-HT women during the first night. (Table 2) 


\section{Group differences on second night}

During the second night, young women had a longer REM latency, more SWS, less WASO and a lower awakening index than other women. In addition, they had a longer TST, higher SE, and less wake time than the postmenopausal groups, as well as less S1 and S2 than the on-HT postmenopausal women. Perimenopausal women had less S1 than on-HT postmenopausal women, and the off-HT group had more SWS than the on-HT group during the second night. (Table 2)

\section{First night effect in combined groups}

When sleep variables were analysed using combined groups, SE increased from the first night of $81.9 \%(1.5 \%)$ to the second night of $87.1 \%(1.0 \%)$. WASO decreased from $66.6(6.0)$ min to 44.6 (4.5) min, and awakening index decreased from $3.22(0.31)$ to $2.20(0.17)$. Sleep latency, S2 latency and REM latency decreased from 20.4 (2.6) min to 15.1 (1.6) min, from 5.5 (0.9) $\mathrm{min}$ to 4.0 (1.3) $\mathrm{min}$, and from 102.3 (6.6) $\mathrm{min}$ to 80.1 (3.7) $\mathrm{min}$, respectively. S1 and S2 percentages increased between nights from $6.5(0.5) \%$ to $10.0(0.5) \%$ and $44.1(0.9)$ $\%$ to 50.1 (1.0) \%, respectively, while the SWS percentage decreased from $25.1(0.8) \%$ to $16.0(0.9) \%$. All these changes were significant at $\mathrm{p}<0.010$.

\section{First night effect in separate groups and group effect}

In group-wise analysis, the amounts of S1 and S2 increased and the amount of SWS decreased in all groups between the first and second nights. The amount of wake time and WASO decreased and SE increased in all groups but not in on-HT postmenopausal women. Further, REM latency decreased in perimenopausal and off-HT postmenopausal women, awakening index decreased in young and off-HT women, and S2 latency decreased in off-HT 
women. (Table 2, Figure 1, Figure 2). In the between-groups Kruskall-Wallis test for the first night effect, no group differences were found in any of the measured variables.

\section{Objective sleep disturbance vs subjective symptoms and hormone profiles}

Perimenopausal women had higher insomnia scores than any other group ( $\mathrm{p}<0.05$ for all). Higher insomnia scores were associated with lower TST and SE, and higher wake and WASO at $\mathrm{p}<0.01$, and higher percentage of $\mathrm{S} 1$ sleep at $\mathrm{p}<0.05$ during both nights. Absolute REM sleep time was lower with higher insomnia scores at $\mathrm{p}<0.01$ during the first night and at $\mathrm{p}<0.05$ during the second night. Further, during the first night, lower REM percentage $(\mathrm{p}<0.01)$ and higher awakening index $(\mathrm{p}<0.05)$ were associated with higher insomnia scores. Higher sleepiness scores were associated with shorter SL during the first, but not the second, night at $\mathrm{p}<0.05$. However, the insomnia or sleepiness scores were not associated with any changes in sleep variables between nights.

Women with more vasomotor symptoms had higher awakening indexes during the first night $(\mathrm{p}<0.01)$. During the second night, they had lower TST and SE ( $p<0.01)$, less S2 and REM sleep $(\mathrm{p}<0.05)$ and more WASO $(\mathrm{p}<0.05)$ and wake time $(\mathrm{p}<0.01)$.

Higher FSH levels were associated with more S2 sleep and WASO $(\mathrm{p}<0.05)$ and less REM sleep $(\mathrm{p}<0.01)$ during the first night. During the second night, higher FSH levels correlated with lower TST and SE and more wake time, WASO and awakenings ( $\mathrm{p}<0.05$ for all). No associations of vasomotor symptoms or FSH levels with inter-night differences were observed.

Insomnia scores and FSH levels were strongly correlated $(\mathrm{p}<0.001)$. Vasomotor symptoms did not associate with insomnia scores or FSH levels.

\section{Discussion}


To our knowledge, our study was the first to evaluate the effect of female reproductive states on first night effect. We found that sleep efficiency increased and WASO and awakenings decreased, thus supporting the idea of an adaptation night in laboratory-based sleep studies. We also discovered an increase in both $\mathrm{S} 1$ and $\mathrm{S} 2$ and a decrease in SWS from the first to the second night, suggesting that the sleep disturbance persists over the second night. The changes were consistent in each group within their own variability limits, albeit less marked sleep consolidation was seen in women using HT.

Previously, SE has been shown to decrease, TST shorten, WASO increase, and SL and REM latency lengthen during a first PSG night in populations aged 2-93 years (Buckley et al., 2013; Curcio et al., 2004; Kis et al., 2014; Moser, Kloesch, Fischmeister, Bauer, \& Zeitlhofer, 2010; Newell et al., 2012; Prihodova et al., 2010; Scholle et al., 2003; Toussaint et al., 1995; Wauquier et al., 1991; Zheng et al., 2012). Tamaki et al 2005) also showed a slower and more fragmented sleep onset during a first night in a sleep laboratory. Lighter sleep during the second night in our study cohort was intriguing, as previously most studies agree on more SWS (Kis et al., 2014; Newell et al., 2012; Scholle et al., 2003; Wauquier et al., 1991; Zheng et al., 2012) and slow wave activity (Tamaki et al., 2005; Toussaint et al., 1995), and less S1 / N1 sleep (Curcio et al., 2004; Scholle et al., 2003) during a second night in a sleep laboratory. However, Buckley et al ( 2013) observed decreased N2 sleep in children, and Curcio et al (2004) increased EEG delta power in adults during a first laboratory night. In our study, changes were uniform and of the same magnitude in all groups. When the proportions of sleep stages were compared, the longer wake time observed during the first night was reduced and replaced by S1 and S2 sleep during the second night. The observed decrease in the proportion of SWS from the first to the second night may therefore be partly due to the increased amount of total sleep. This suggests that a laboratory environment may 
in fact disturb the lighter stages of sleep more whereas SWS deprivation is more negligible. Therefore, there would be no necessity to increase the amount of SWS, but rather to increase the total amount of sleep. The finding poses a question on whether one adaptation night is enough. A previous report by Le Bon et al ( 2001) suggests that this may be true. In their study group of 26 young volunteers, the effects of a first night on NREM sleep lasted for one night, but REM sleep power bands increased and REM latencies decreased up until the fourth night. The different result profile may be affected by the age difference and the fact that their cohort was half male.

In our study, sleep in young women was deeper and more consolidated than in other groups, but in the peri- and postmenopausal women it was quite similar. At least three large population-based studies have addressed the effects of midlife reproductive state on objective sleep quality (Hachul et al., 2010; Hachul et al., 2015; Young et al., 2003), showing that periand postmenopausal women have more SWS and less REM sleep than premenopausal women, even when adjusting for age. Sleep in postmenopausal HT users is less fragmented and less prone to external disturbance than sleep in non-users (Hachul et al., 2010; Moe et al., 2001; Young et al., 2003). Further, according to our results higher serum FSH concentrations are associated with more fragmented sleep. Symptomatic perimenopausal women also have worse sleep quality that is improved by HT (Polo-Kantola, 2011; Shaver et al., 1988; Young et al., 2003), which is in line with our observation on more fragmented sleep in women with more vasomotor symptoms. However, HT users have more S1 sleep and less SWS than nonHT-users (Young et al., 2003). This agrees with our findings of less SWS in HT users compared to non-users during both nights. Lighter sleep in on-HT women might be interpreted as a deteriorative effect of HT on sleep. However, since the use of HT was selfchosen, it is more likely that HT users were initially more symptomatic than off-HT women. 
Therefore, their sleep could be more vulnerable for outside stressors, being beyond the alleviating effect of HT.

Subjective and objective sleep disturbances correlate less in postmenopausal women than in men or premenopausal women, especially in symptomatic women (Vitiello, Larsen, \& Moe, 2004; Young et al., 2003). In HT users, the correlation between objective and subjective sleep quality is negligible (Vitiello et al., 2004). As insomnia scores varied between groups, we further estimated the possible association of subjective sleep disturbance with sleep variables. Our sample size did not allow for reliable group-wise correlation analyses, but groups combined, complaints of insomnia were associated with lighter and more fragmented sleep, thus linking subjective and objective sleep disturbance together. Furthermore, as some insomnia patients seem to exhibit a reverse first night effect (RFNE) (Hauri \& Olmstead, 1989; Riedel, Winfield, \& Lichstein, 2001), we looked for correlations between insomnia scores and the observed first night effect, but no associations were observed. This suggests that RFNE did not affect our results.

There are some limitations in our study. First, only central EEG electrodes were used in young women due to limited resources at the Helsinki study site. This limits the assessment of posterior alpha activity with possible difficulties in the differentiation between wake and drowsiness (Santamaria \& Chiappa, 1987). However, as their EEG patterns were clear, it very unlikely formed any error in the sleep stage analysis. Second, since the sleep data were collected and analysed between 2001 and 2004, the sleep stage analysis was performed using the Rechtschaffen and Kales criteria (Rechtschaffen \& Kales, 1968), instead of the AASM criteria (Iber, Ancoli-Israel, Chesson, \& Quan, 2007). This may lead to overestimation of S2/N2 sleep and underestimation of S1/N1 sleep and SWS in our data (Moser et al., 2009). 
As the difference was consistent across groups and nights, it unlikely formed any error to the interpretation. The long data gathering time also made it impossible to control for ambient light or time of year. Also, sleep macroarchitechture may not be a sensitive enough marker, and analysing sleep microarchitecture might have given additional information. Third, while we excluded women with previous diagnosis of sleep apnea and restless legs syndrome, we did not use leg electrodes or nasal pressure transducers. Therefore, undiagnosed diseases could not be ruled out. Fourth, all young women used oral contraceptives, which have some, albeit divergent, effects on sleep (Baker, Mitchell, \& Driver, 2001; Hachul et al., 2010) and thus their results are possibly not comparative with women not using contraceptives. Fifth, the women in our study were relatively healthy, and therefore, the results cannot be generalised to a general population. Last, as is typical in PSG studies, our patient groups are small and uneven, which decreases statistical power and may limit the possibilities to detect group differences.

\section{Conclusion}

Our study shows a distortion of PSG measured sleep of women in the first night in sleep laboratory. This distortion is prolonged to the second night as lighter objective sleep. Regardless of age-mediated changes in sleep architecture, a first night in a sleep laboratory produces very consistent changes in both sleep consolidation and the proportions of sleep stages in women of different ages. This change appears to be unaffected by menopausal status or HT use, nor is it influenced by subjective complaints of insomnia. 


\section{References}

Agnew, H., Webb, W., \& Williams, R. (1966). The first night effect: an EEG study of sleep. Psychophysiology, 2, 263-266.

Baker, F., Mitchell, D., \& Driver, H. (2001). Oral contraceptives alter sleep and raise body temperature in young women. Pflügers Archiv, 424, 729-737.

Buckley, A., Wingert, K., Swedo, S., Thurm, A., Sato, S., Appel, S., et al. (2013). First Night Effect Analysis in a Cohort of Young Children with Autism Spectrum Disorder. Journal of Clinical Sleep Medicine, 9(1), 67-70.

Curcio, G., Ferrara, M., Piergianni, A., Fratello, F., \& De Gennaro, L. (2004). Paradoxes of the first-night effect: a quantitative analysis of antero-posterior EEG topography. Clinical Neurophysiology, 115, 1178-1188.

de Villiers, T., Pines, A., Panay, N., Gambacciani, M., Archer, D., Baber, R., et al. (2013). Updated 2013 International Menopause Society recommendations on menopausal hormone therapy and preventive strategies for midlife health. Climacteric, 16(3), 316317.

Hachul, H., Andersen, M., Bittencourt, L., Santos-Silva, R., Conway, S., \& Tufik, S. (2010). Does the reproductive cycle influence sleep patterns in women with sleep complaints? Climacteric, 13, 594-603.

Hachul, H., Frange, C., Bezerra, A., Hirotsu, C., Pires, G., Andersen, M., et al. (2015). The effect of menopause on objective sleep parameters: Data from an epidemiologic study in São Paulo, Brazil. Maturitas, 80, 170-178.

Hauri, P., \& Olmstead, E. (1989). Reverse first night effect in insomnia. Sleep, 12(2), 97-105.

Iber, C., Ancoli-Israel, S., Chesson, A., \& Quan, S. (2007). The AASM manual for the scoring of sleep and associated events: rules, terminology, and technical specifications, 1 st ed. Westchester, Illinois: American Academy of Sleep Medicine.

Kalleinen, N., Polo, O., Himanen, S.-L., Joutsen, A., \& Polo-Kantola, P. (2008). The effect of estrogen plus progestin treatment on sleep: a randomized, placebo-controlled, doubleblind trial in premenopausal and late postmenopausal women. Climacteric, 11, 233243.

Kalleinen, N., Polo, O., Himanen, S.-L., Joutsen, A., Urrila, A., \& Polo-Kantola, P. (2006). Sleep deprivation and hormone therapy in postmenopausal women. Sleep Medicine, 7(5), 436-447.

Kis, A., Szakadát, S., Simor, P., Gombos, F., Horváth, K., \& Bódizs, R. (2014). Objective and Subjective Components of the First-Night Effect in Young Nightmare Sufferers and Healthy Participants. Behavioral Sleep Med, 12(6), 469-480.

Lampio, L., Polo-Kantola, P., Polo, O., Kauko, T., Aittokallio, J., \& Saaresranta, T. (2014). Sleep in midlife women: effects of menopause, vasomotor symptoms, and depressive symptoms. Menopause, 21(11), 1217-1224.

Le Bon, O., Staner, L., hofmann, G., Dramaix, M., San Sebastian, I., Murphy, J., et al. (2001). The first-night effect may last more than one night. Journal of Psychiatric Research, 35, 165-172.

Lorenzo, J.-L., \& Barbanoj, M.-J. (2002). Variability of sleep parameters across multiple laboratory sessions in healthy young subjects: The "very first night effect". Psychophysiology, 39, 409-413.

Moe, K., Larsen, L., Vitiello, M., \& Prinz, P. (2001). Estrogen Replacement Therapy Moderates the Sleep Disruption Associated with Nocturnal Blood Sampling. Sleep, 24(8), 886-894. 
Moser, D., Anderer, P., Gruber, G., Parapatics, S., Loretz, E., Boeck, M., et al. (2009). Sleep classification according to AASM and Rechtschaffen \& Kales: effects on sleep scoring parameters. Sleep, 32(2), 139-149.

Moser, D., Kloesch, G., Fischmeister, F., Bauer, H., \& Zeitlhofer, J. (2010). Cyclic alternating pattern and sleep quality in healthy subjects-Is there a first-night effect on different approaches of sleep quality? . Biological Phychology, 83, 20-26.

Newell, J., Mairesse, O., Verbanck, P., \& Neu, D. (2012). Is a one-night stay in the lab really enough to conclude? First-night effect and night-to-night variability in polysomnographic recordings among different clinical population samples. Psychiatry Research, 200, 795-801.

Partinen, M., \& Gislason, T. (1995). Basic Nordic Sleep Questionnaire (BNSQ): a quantitated measure of subjective sleep complaints. Journal of Sleep Research, 4 Suppl 1, 150155.

Polo-Kantola, P. (2011). Sleep probems in midlife and beyond. Maturitas, 86, 224-232.

Prihodova, I., Paclt, I., Kemlink, D., Skibova, J., Ptacek, R., \& Nevsimalova, S. (2010). Sleep disorders and daytime sleepiness in children with attention-deficit/hyperactivity disorder: A two-night polysomnographic study with a multiple sleep latency test. Sleep Medicine, 11, 922-928.

Rechtschaffen, A., \& Kales, A. (1968). A manual of standardized terminology, techniques and scoring system for sleep stages of human subjects. Los Angeles, California: Brain Information Service/Brain Research Institute, University of California.

Riedel, B., Winfield, C., \& Lichstein, K. (2001). First night effect and reverse first night effect in older adults with primary insomnia: does anxiety play a role? Sleep Medicine, 2, 125-133.

Santamaria, J., \& Chiappa, K. (1987). The EEG of drowsiness in normal adults. Journal of Clinical Neurophysiology, 4(4), 327-382.

Scholle, S., Scholle, H.-C., Kemper, A., Glaser, S., Rieger, B., Kemper, G., et al. (2003). First night effect in children and adolescents undergoing polysomnography for sleepdisordered breathing. Clinical Neurophysiology, 114, 2138-2145.

Shaver, J., Giblin, E., Lentz, M., \& Lee, K. (1988). Sleep patterns and stability in perimenopausal women. Sleep, 11, 556-561.

Tamaki, M., Nittono, H., Hayashi, M., \& Hori, T. (2005). Examination of the First-Night Effect during the Sleep-Onset Period. Sleep, 28(2), 195-202.

Toussaint, M., Luthringer, R., Schaltenbrand, N., Carelli, G., Lainey, E., Jacqmin, A., et al. (1995). First-Night Effect in Normal Subjects and Psychiatric Inpatients. Sleep, 18(6), 463-469.

Wauquier, A., van Sweden, B., Kerkhof, G., \& Kamphuisen, H. (1991). Ambulatory first night sleep effect recording in the elderly. Behavioural Brain Research, 42, 7-11.

Webb, W., \& Campbell, S. (1979). The first night effect revisited with age as a variable. Waking and Sleeping, 3(4), 319-324.

Vitiello, M., Larsen, L., \& Moe, K. (2004). Age-related sleep change: Gender and estrogen effects on the subjective-objective sleep quality relationships of healthy, noncomplaining older men and women. Journal of Psychosomatic Research, 56, 503510.

Young, T., Rabago, D., Zgierska, A., Austin, D., \& Finn, L. (2003). Objective and Subjective Sleep Quality in Premenopausal, Perimenopausal, and Postmenopausal Women in the Wisconsin Sleep Cohort Study. Sleep, 26(6), 667-672.

Zheng, H., Sowers, M., Buysse, D., Consens, F., Kravitz, H., Matthews, K., et al. (2012). Sources of Variability in Epidemiological Studies of Sleep Using Repeated Nights of 
In-Home Polysomnography: SWAN Sleep Study. Journal of Clinical Sleep Medicine, 8(1), 87-96. 


\section{Acknowledgements}

We are grateful to Dr Tarja Porkka-Heiskanen for the general arrangements of the study and the support of the data collection in Helsinki.

The study was financially supported by a European Commission Grant (QLK6-CT-2000-00499), and grants from the Väinö and Laina Kivi Foundation (P.P-K.), The Finnish Menopause Society Foundation (P.P-K.), The Finnish Medical Foundation (P.P-K. and N.K.), The Turku University Foundation (P.P-K., N.K. and I.V.), The Finnish Academy (A.S.U.), and by a Finnish Government grant (N.K.) 
TST (minutes)

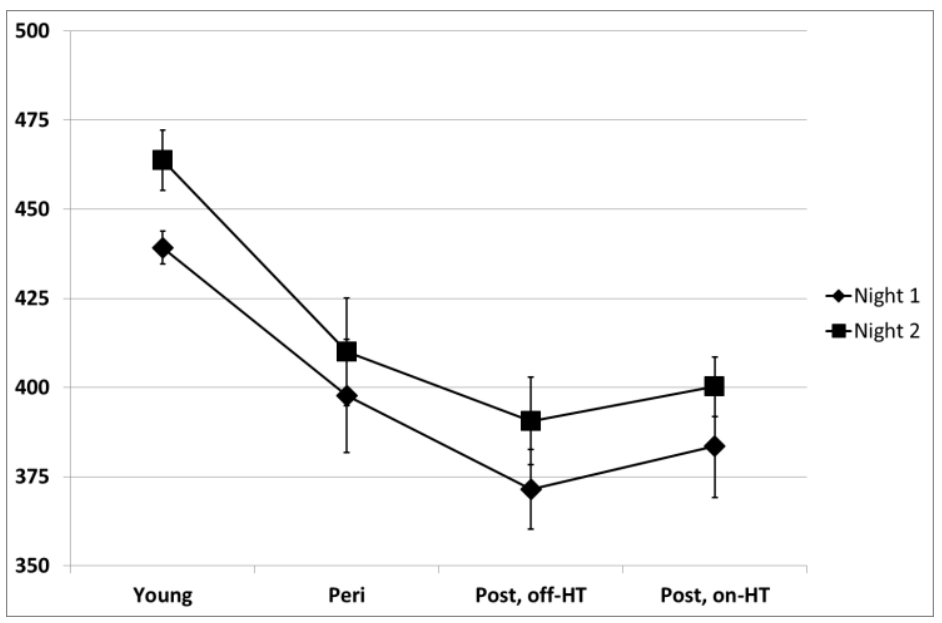

SE $(\%)$

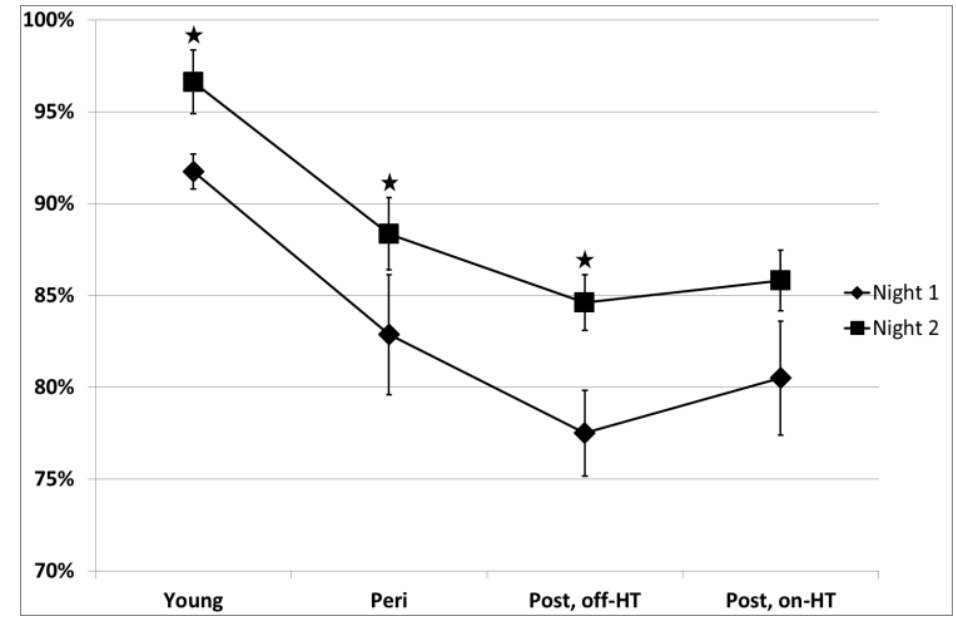

WASO (minutes)

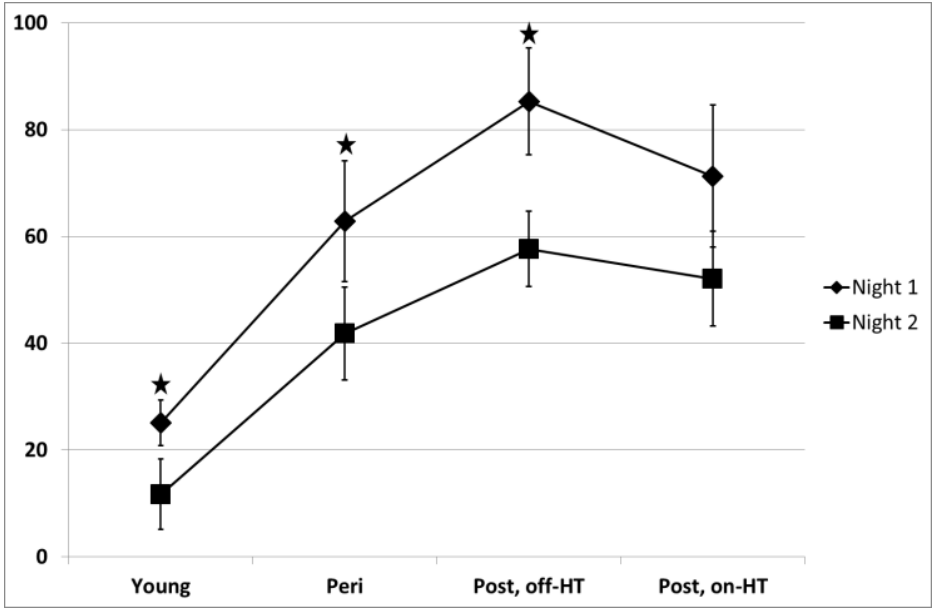

Awakening index (events/h) 


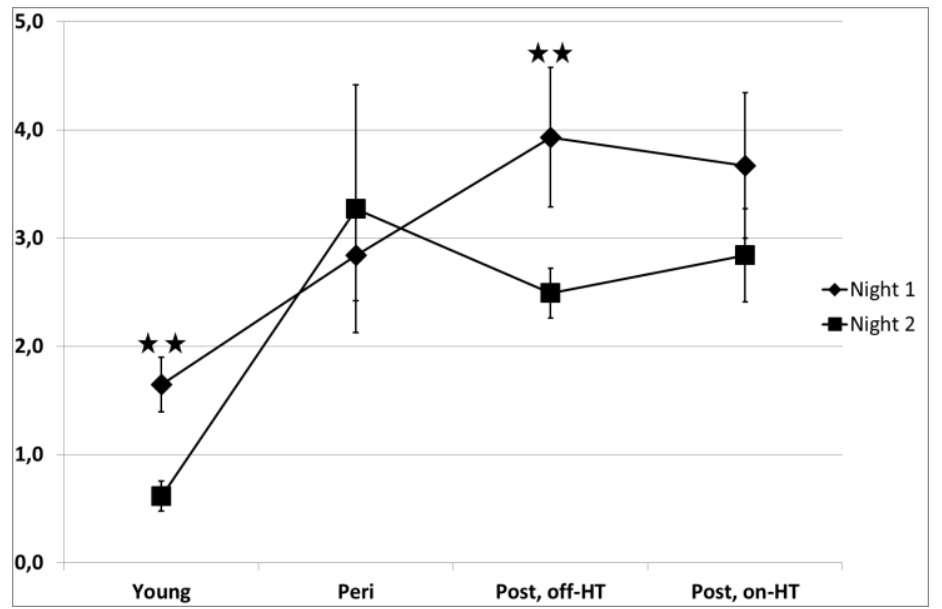

Figure 1. Sleep consolidation during study nights in the groups. Error bars in SEM. $\star p<0.05, \star \star$ $\mathrm{p}<0.01$ between nights. 
S1 sleep (\% of TST)

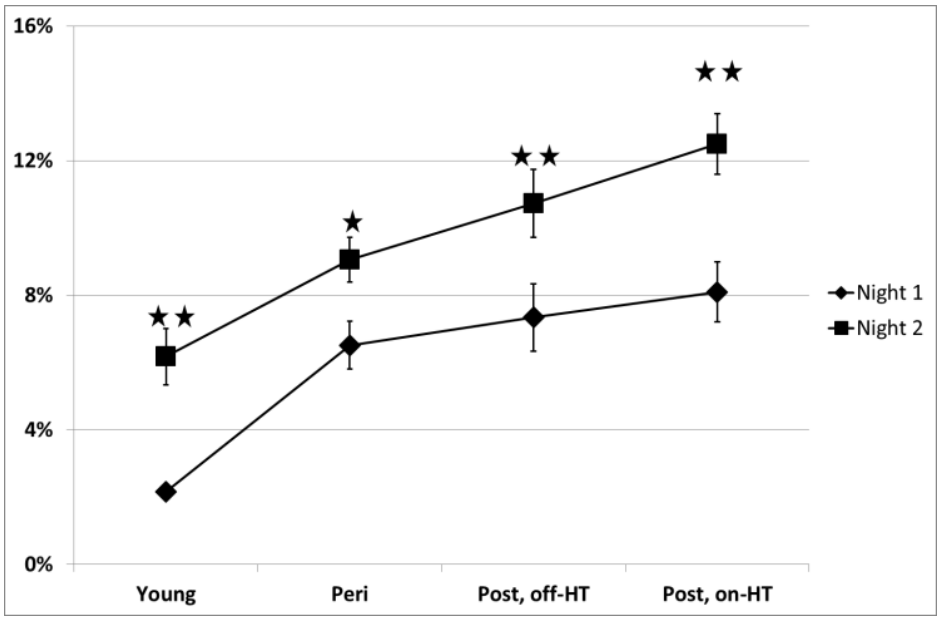

S2 sleep (\% of TST)

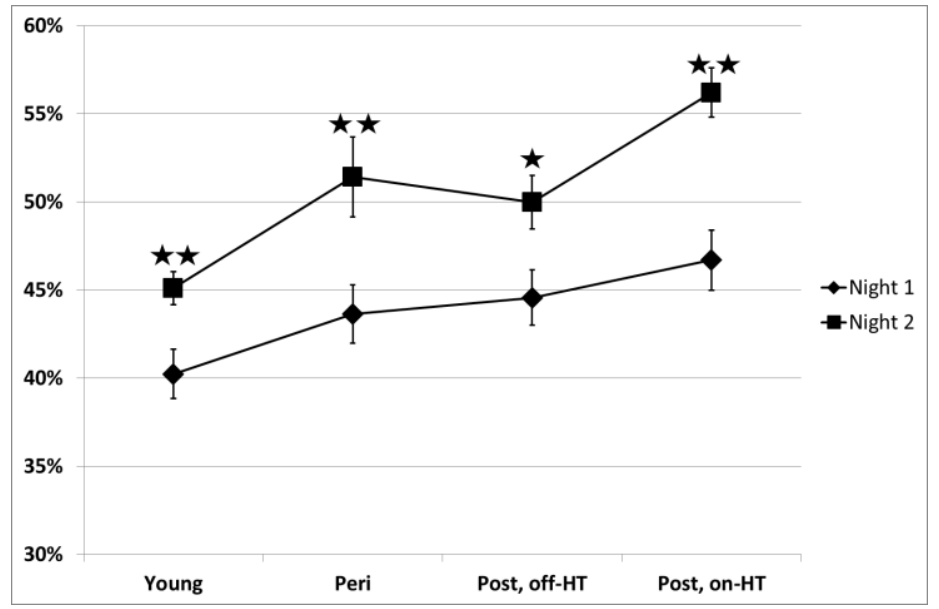

SWS (\% of TST)

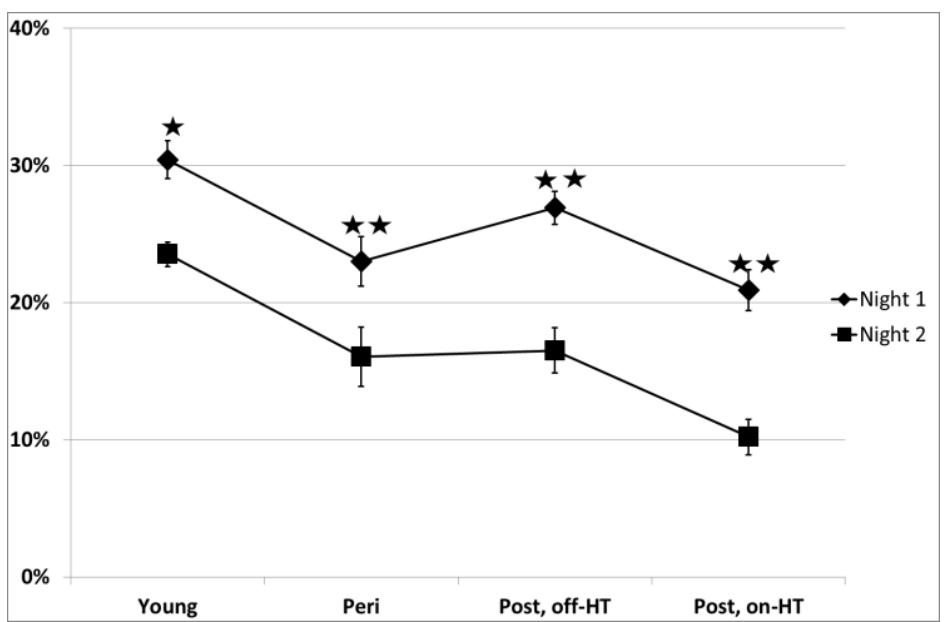

REM latency (minutes) 


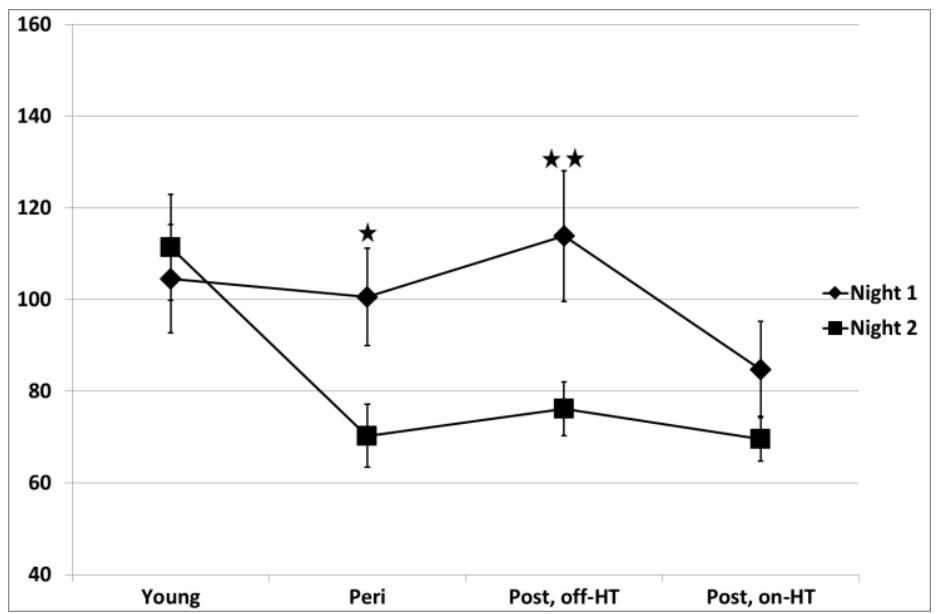

Figure 2. Sleep stage indexes during study nights in the groups. Error bars in SEM. $\star p<0.05, \star \star$ $\mathrm{p}<0.01$ between nights. 
Table 1. Baseline data of the study groups.

\begin{tabular}{|c|c|c|c|c|c|c|c|c|c|c|c|c|c|c|}
\hline & Age (y) & Parity $(\mathrm{N})$ & $\begin{array}{c}\mathrm{BMI} \\
\left(\mathrm{kg} / \mathrm{m}^{2}\right)\end{array}$ & & $\begin{array}{c}\mathrm{FSH}(\mathrm{U} / \mathrm{I}) \\
\mathrm{a}\end{array}$ & & $\mathrm{E} 2(\mathrm{nmol} / \mathrm{l})$ & & $\begin{array}{c}\text { Insomnia } \\
\text { score a }\end{array}$ & & $\begin{array}{l}\text { Sleepiness } \\
\text { score } a\end{array}$ & $\begin{array}{l}\text { Vasomotor } \\
\text { symptom } \\
\text { score a }\end{array}$ & $\begin{array}{c}\text { BDI } \\
\text { score }\end{array}$ & \\
\hline Young women & $23.1 \pm 0.5$ & 0 & $22.4 \pm 0.7$ & ** & $3.5 \pm 0.8$ & ** & $0.08 \pm 0.02$ & $\S$ & $10.0 \pm 0.7$ & ** & $10.3 \pm 0.7$ & $2,6 \pm 0,3$ & $1,5 \pm 0,5$ & ** \\
\hline Perimenopausal women & $48.0 \pm 0.4$ & $1,5 \pm 0,3$ & $24.2 \pm 0.6$ & * & $11.4 \pm 1.3$ & ** & $0.33 \pm 0.09$ & $* *$ & $13.2 \pm 1.0$ & * & $9.8 \pm 1.0$ & $2,6 \pm 0,2$ & $3,9 \pm 0,8$ & \\
\hline Off-HT postmenopausal women & $63.4 \pm 0.8$ & $2,4 \pm 0,2$ & $27.4 \pm 1.1$ & & $78.9 \pm 7.1$ & & $0.03 \pm 0.00$ & & $16.9 \pm 0.6$ & & $11.0 \pm 0.7$ & $3,7 \pm 0,5$ & $6,9 \pm 0,9$ & \\
\hline On-HT postmenopausal women & $63.1 \pm 0.9$ & $1,7 \pm 0,2$ & $24.9 \pm 0.6$ & & $13.8 \pm 4.0$ & ** & $0.19 \pm 0.03$ & * & $13.4 \pm 1.2$ & * & $11.8 \pm 1.4$ & $2,6 \pm 0,3$ & $4,2 \pm 0,9$ & \\
\hline
\end{tabular}

$* \mathrm{p}<0.05, * * \mathrm{p}<0.01$ compared to off-HT postmenopausal women

$\S \mathrm{p}<0.05$ compared to perimenopausal women

$\mathrm{BDI}=$ Beck Depression Inventory

a Correlated with sleep variables. 
Table 2. Sleep indexes in study groups.

\begin{tabular}{|c|c|c|c|c|c|c|c|c|c|c|c|c|c|c|}
\hline & \multicolumn{2}{|c|}{ Young, $n=10$} & \multicolumn{3}{|c|}{ Perimenopausal, $\mathrm{n}=15$} & & \multicolumn{3}{|c|}{ Postmenopausal, off-HT, $n=22$} & & \multicolumn{3}{|c|}{ Postmenopausal, on-HT, $\mathrm{n}=14$} & \\
\hline & Night 1 & Night 2 & Night 1 & & Night 2 & & Night 1 & & Night 2 & & Night 1 & & Night 2 & \\
\hline & Mean \pm SEM & Mean \pm SEM & Mean \pm SEM & & Mean \pm SEM & & Mean \pm SEM & & Mean \pm SEM & & Mean \pm SEM & & Mean \pm SEM & \\
\hline Total sleep time min & $439.2 \pm 4.6$ & $448.1 \pm 9.0$ & $397.7 \pm 15.9$ & & $410.1 \pm 10.8$ & & $371.4 \pm 11.2$ & ** & $389.1 \pm 8.7$ & ** & $383.5 \pm 14.3$ & * & $400.2 \pm 8.4$ & ** \\
\hline Sleep efficiency of time analysed \% & $91.7 \pm 1.0$ & $94.7 \pm 1.6$ & $82.9 \pm 3.3$ & & $88.0 \pm 2.0$ & & $77.5 \pm 2.3 \%$ & ** & $84.0 \pm 1.6$ & ** & $80.5 \pm 3.1$ & & $85.8 \pm 1.7$ & ** \\
\hline Sleep latency min & $14.6 \pm 3.0$ & $14.6 \pm 3.0$ & $19.4 \pm 6.9$ & & $14.1 \pm 3.1$ & & $22.6 \pm 4.0$ & & $16.5 \pm 3.1$ & & $22.2 \pm 5.4$ & & $14.5 \pm 3.4$ & \\
\hline S2 latency from sleep onset min & $18.5 \pm 2.7$ & $2.8 \pm 1.4$ & $25.7 \pm 6.8$ & & $4.5 \pm 3.0$ & & $28.2 \pm 4.1$ & & $3.2 \pm 1.8$ & & $5.6 \pm 1.7$ & & $5.7 \pm 3.6$ & \\
\hline SWS latency from sleep onset min & $24.9 \pm 3.3$ & $12.8 \pm 2.1$ & $37.5 \pm 8.2$ & & $20.0 \pm 4.0$ & & $42.8 \pm 6.9$ & & $18.0 \pm 3.8$ & & $14.5 \pm 2.6$ & & $23.3 \pm 5.2$ & \\
\hline REM latency from sleep onset min & $104.5 \pm 11.8$ & $111.4 \pm 11.5$ & $100.5 \pm 10.6$ & & $74.9 \pm 5.4$ & * & $113.8 \pm 14.2$ & & $76.2 \pm 5.8$ & * & $84.7 \pm 10.5$ & & $69.6 \pm 4.9$ & * \\
\hline S1 as $\%$ of TST & $2.2 \pm 0.2$ & $6.4 \pm 0.8$ & $6.5 \pm 0.7$ & ** & $9.0 \pm 0.7 \%$ & & $7.3 \pm 1.0$ & ** & $10.6 \pm 0.9$ & & $8.1 \pm 0.9$ & ** & $12.5 \pm 0.9$ & ${ }^{* *} \#$ \\
\hline S2 as $\%$ of TST & $40.2 \pm 1.4$ & $46.7 \pm 1.0$ & $43.6 \pm 1.7$ & & $51.1 \pm 2.3$ & & $44.6 \pm 1.6$ & & $50.0 \pm 1.6$ & & $46.7 \pm 1.7$ & & $56.2 \pm 1.4$ & ** \\
\hline SWS as $\%$ of TST & $30.4 \pm 1.4$ & $24.4 \pm 1.0$ & $23.0 \pm 1.8$ & * & $15.5 \pm 1.8$ & $* *$ & $26.9 \pm 1.2$ & & $16.2 \pm 1.5$ & ** & $20.9 \pm 1.5$ & ${ }^{* *} \S$ & $10.2 \pm 1.3$ & ${ }^{* *} \S$ \\
\hline REM as $\%$ of TST & $27.2 \pm 1.6$ & $22.6 \pm 1.3$ & $26.8 \pm 1.5$ & & $24.4 \pm 1.6$ & & $21.2 \pm 1.2$ & ${ }^{*} \#$ & $23.3 \pm 1.2$ & & $24.3 \pm 1.3$ & & $21.0 \pm 1.0$ & \\
\hline Wake after sleep onset min & $25.1 \pm 4.3$ & $10.5 \pm 6.6$ & $62.8 \pm 11.3$ & & $41.5 \pm 8.8$ & * & $85.3 \pm 10.0$ & ** & $57.5 \pm 7.0$ & ** & $71.3 \pm 13.4$ & * & $52.1 \pm 8.9$ & ** \\
\hline $\mathrm{S} 1 \mathrm{~min}$ & $9.5 \pm 0.9$ & $28.5 \pm 3.9$ & $25.6 \pm 2.8$ & $* *$ & $36.5 \pm 2.7$ & & $26.1 \pm 2.7$ & $* *$ & $41.2 \pm 3.6$ & & $30.3 \pm 3.0$ & ** & $50.2 \pm 3.7$ & ** \\
\hline $\mathrm{S} 2 \mathrm{~min}$ & $176.4 \pm 5.2$ & $209.4 \pm 6.5$ & $172.0 \pm 7.6$ & & $209.6 \pm 11.1$ & & $166.2 \pm 7.9$ & & $194.1 \pm 7.3$ & & $178.3 \pm 7.8$ & & $225.3 \pm 8.2$ & \\
\hline SWS min & $133.6 \pm 6.1$ & $108.9 \pm 4.2$ & $92.0 \pm 8.5$ & $* *$ & $62.9 \pm 6.6$ & $* *$ & $99.0 \pm 4.8$ & ** & $63.6 \pm 6.4$ & $* *$ & $79.9 \pm 5.9$ & ** & $40.4 \pm 5.2$ & ** \\
\hline REM min & $119.8 \pm 7.7$ & $101.4 \pm 6.6$ & $108.2 \pm 8.6$ & & $100.9 \pm 8.2$ & & $80.1 \pm 5.5$ & ** & $90.3 \pm 5.1$ & & $95.0 \pm 8.1$ & & $84.4 \pm 4.5$ & \\
\hline Wake min & $39.6 \pm 4.6$ & $25.0 \pm 7.3$ & $82.2 \pm 15.7$ & & $55.7 \pm 9.3$ & & $107.9 \pm 11.3$ & ** & $73.9 \pm 7.3$ & ** & $93.5 \pm 14.9$ & & $66.6 \pm 8.3$ & ** \\
\hline Awakening index & $1.64 \pm 0.25$ & $0.61 \pm 0.14$ & $2.84 \pm 0.42$ & & $2.18 \pm 0.23$ & $* *$ & $3.93 \pm 0.65$ & ** & $2.51 \pm 0.22$ & $* *$ & $3.67 \pm 0.67$ & * & $2.84 \pm 0.43$ & ** \\
\hline
\end{tabular}

$* \mathrm{p}<0.05, * * \mathrm{p}<0,01$ compared to young women

\# $\mathrm{p}<0.05$ compared to perimenopausal women

$\S \mathrm{p}<0.05$ compared to off-HT postmenopausal women 